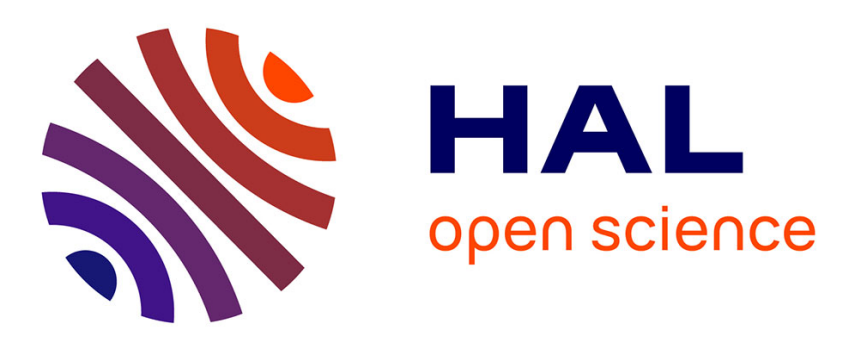

\title{
On the use of polynomial chaos expansions and generalized moments within the frame of gas radiation in non-uniform media
}

\author{
Frédéric André, Mathieu Galtier
}

\section{- To cite this version:}

Frédéric André, Mathieu Galtier. On the use of polynomial chaos expansions and generalized moments within the frame of gas radiation in non-uniform media. Journal of Quantitative Spectroscopy and Radiative Transfer, 2015, 164, pp.184-192. 10.1016/j.jqsrt.2015.06.005 . hal-02064943

\section{HAL Id: hal-02064943 https://hal.science/hal-02064943}

Submitted on 23 Apr 2019

HAL is a multi-disciplinary open access archive for the deposit and dissemination of scientific research documents, whether they are published or not. The documents may come from teaching and research institutions in France or abroad, or from public or private research centers.
L'archive ouverte pluridisciplinaire HAL, est destinée au dépôt et à la diffusion de documents scientifiques de niveau recherche, publiés ou non, émanant des établissements d'enseignement et de recherche français ou étrangers, des laboratoires publics ou privés. 


\section{ON THE USE OF POLYNOMIAL CHAOS EXPANSIONS AND GENERALIZED MOMENTS WITHIN THE FRAME OF GAS RADIATION IN NON-UNIFORM MEDIA.}

Frédéric André*, Mathieu Galtier

Université de Lyon, CNRS

INSA-Lyon, CETHIL, UMR5008, F-69621, Villeurbanne, France

Université Lyon 1, CETHIL, UMR5008, F-69622, France 


\begin{abstract}
In many applications involving gaseous media at high temperature, accurate but computationally efficient models are required for the radiative properties of gases. The aim of the present work is to show how, by combining results from Polynomial Chaos framework and Devyatov's Method of Moments, one can provide simple estimates of the transmission functions of gases both in uniform and non-uniform media. The proposed model only involves polynomials. It is probably one of the simplest in terms of mathematical formulation but also one of the most sophisticated, considering the concepts that it involves. It can be applied in uniform and non-uniform media, where it is shown to be equivalent, in terms of accuracy, to usual C- $k$ models.
\end{abstract}

KEYWORDS: gas radiation, polynomial chaos, generalized moments, carbon dioxide, Cutteridge-Devyatov polynomials.

\title{
1. INTRODUCTION
}

Radiative heat transfer in gaseous media arises in many situations. Gases may be at high temperature, as in combustion applications, or cold, as in some atmospheric studies. The most accurate model to estimate the radiative properties of gases is the so-called Line-By-Line (LBL) approach [1,2]. It consists in calculating gas absorption spectra at high resolution directly from spectroscopic databases. Nevertheless, it is too computationally expensive to be considered in many cases (such as in multi-physic or three-dimensional problems) for which more computationally efficient approaches are required.

Many approximate models were proposed during the past decades. Most of them (especially those based on the so-called $k$-distribution approach, [3]) achieve LBL accuracy in uniform situations. Usually, their main source of error comes from the approximate treatment of nonuniformities that requires additional assumptions, such as the scaling or correlation of 
absorption spectra in various thermophysical states. In most situations, those assumptions provide accurate results, as soon as small temperature gradients are found inside the gas. When higher gradients are involved, recourse to more sophisticated techniques, such as the Multi-Group [4,5], Fictitious gases [6] or Multi-spectral [7,8] approximations, is required.

Recently, the generalized $k$-moment approach $[9,10]$ was proposed to estimate cumulative distribution functions directly from LBL data. The method enables to represent those functions as sums of polynomials of a certain type: the so-called Cutteridge-Devyatov polynomials. It was found to be accurate both for the calculation of distribution functions and for applications in radiative heat transfer (results, in terms of narrow band averaged spectra, are provided in the case of $\mathrm{H}_{2} \mathrm{O}$ in Ref. [10]).

The Generalized $k$-moment approach is based on Devyatov's Method of Moments (DMM) described in Refs. [11,12]. This method is similar to the so-called Polynomial Chaos (PC) technique proposed in 1938 by Wiener [13], but introduced in a slightly different way. Some theoretical links between the two approaches are discussed in this paper (additional details are also provided in Appendix).

The aim of the present work is to show how, by combining results from PC and DMM, it is possible to propose simple and accurate models for the radiative properties of gases in uniform and non-uniform media. It is organized as follows: 1/ in the first section, the method is introduced by considering the problem of estimating a scaling function between spectra in two distinct thermophysical states; 2 / the second part is dedicated to a short description of the PC framework; $3 /$ then, in the third part, it is shown how it is possible to replace the implicit equation associated to the scaling assumption, which is highly non linear, by a simple polynomial one from the use of concepts taken from PC theory. Explicit formulas are also given to estimate transmission spectra as polynomials; 4/ the fourth, and final section, describes some results of comparisons of the proposed Cutteridge-Devyatov Polynomial 
Chaos (CDPC) model and reference Line-By-Line calculations. CDPC approximation is shown to provide results as accurate as usual $k$-distribution approaches at a very low computational cost (polynomials at orders 10 up to 14) in uniform and non-uniform situations.

\section{PRINCIPLE OF THE METHOD.}

In order to introduce our methodology, we are going to study the following problem. We consider a spectral band $\Delta \eta$ over which: 1/ the Planck function is constant (we thus restrict here our approach to narrow band models), 2/ the absorption coefficient in any thermophysical state takes strictly positive values (no transparency region of the gas is inside the interval). We define a reference length that will be from now on written $L^{\text {ref }}$ and two thermophysical states. They are represented as vectors $\underline{\phi}$ whose components are the temperature, total pressure and composition of the gas associated to each state. One of them will be written $\underline{\phi}^{\text {ref }}$, and will play the role of a reference, and the second one $\underline{\phi}$. Gas spectra are assumed to be scaled (as defined by Modest in Ref. [3] - see Eq. (30) from this reference) which means that we can find a real $u$, that depends implicitly on the state vectors $\underline{\phi}^{\text {ref }}$ and $\underline{\phi}$ such that:

$$
\frac{1}{\Delta \eta} \cdot \int_{\Delta \eta} \exp \left[-\kappa_{\eta}(\underline{\phi}) \cdot L^{r e f}\right] d \eta=\frac{1}{\Delta \eta} \cdot \int_{\Delta \eta} \exp \left[-u \cdot \kappa_{\eta}\left(\underline{\phi}^{r e f}\right) \cdot L^{r e f}\right] d \eta
$$

Our objective is to find $u$.

Let us assume that the solution $u$ to Eq. (1) lies inside some bounded interval, $\left[u_{\min }, u_{\max }\right]$ with $u_{\max }>u_{\min }>0$ (this obviously restricts the approach to spectral bands over which the gas is not transparent, as assumed previously). If we have no a priori information about the solution, then the range $\left[u_{\min }, u_{\max }\right]$ extends over several orders of magnitude (due to the 
behavior of absorption spectra). Accordingly, we normalize the search space over which we are going to seek $u$ by introducing a new variable, that will be written $\xi$, such that:

$$
u=u(\xi)=u_{\min } \cdot\left(\frac{u_{\max }}{u_{\min }}\right)^{\xi}, \xi \in[0,1]
$$

In order to introduce the concept of Polynomial Chaos in a simple way, let us describe how the problem set by Eq. (1) could be handled by application of a Stochastic Optimization Technique. Using this kind of methods to solve Eq. (1) would consist in the following steps [14]:

$1 /$ for a prescribed integer number $N$, choose inside the interval $[0,1] N$ random numbers $\xi_{1}, \ldots, \xi_{N}$ according to a uniform probability distribution. Then, the objective function $F(\xi)$ defined as (from Eq. (1)):

$$
\begin{aligned}
F(\xi) & =\frac{1}{\Delta \eta} \cdot \int_{\Delta \eta} \exp \left[-u(\xi) \cdot \kappa_{\eta}\left(\underline{\phi}^{r e f}\right) \cdot L^{r e f}\right] d \eta-\frac{1}{\Delta \eta} \cdot \int_{\Delta \eta} \exp \left[-\kappa_{\eta}(\underline{\phi}) \cdot L^{r e f}\right] d \eta \\
& =\tau^{\Delta \eta}\left[L(\xi), \underline{\phi}^{r e f}\right]-\tau^{\Delta \eta}\left(L^{r e f}, \underline{\phi}\right)
\end{aligned}
$$

where:

$$
\tau^{\Delta \eta}\left[L(\xi), \underline{\phi}^{r e f}\right]=\frac{1}{\Delta \eta} \cdot \int_{\Delta \eta} \exp [-\kappa_{\eta}\left(\underline{\phi}^{r e f}\right) \cdot \underbrace{u(\xi) \cdot L^{r e f}}_{L(\xi)}] d \eta
$$

and:

$$
\tau^{\Delta \eta}\left(L^{r e f}, \underline{\phi}\right)=\frac{1}{\Delta \eta} \cdot \int_{\Delta \eta} \exp \left[-\kappa_{\eta}(\underline{\phi}) \cdot L^{r e f}\right] d \eta
$$

can be evaluated to provide $N$ values of $F: F\left(\xi_{1}\right), \ldots, F\left(\xi_{N}\right)$.

2/ The next step is usually to search the integer index $i \in\{1, \ldots, N\}$ that corresponds to the minimum of the absolute value of $F\left(\xi_{i}\right), \xi_{i} \in\left\{\xi_{1}, \ldots, \xi_{N}\right\}$. This index is associated to the best estimate, inside the set $\left\{\xi_{1}, . ., \xi_{N}\right\}$, of the solution to Eq. (1). 
3/ If the value of $F\left(\xi_{i}\right)$ is lower than a user-defined threshold, then stop the process. Otherwise, apply the evolutionary process associated to the chosen Stochastic Optimization Technique (by combining/mutating genes in the case of Genetic Algorithms, or by generating a direction of propagation for Particle Swarm techniques, etc) and go back to step 1.

The task of the evolutionary scheme is to decrease, at each step of the process, the size of the search space. The method is thus iterative and continues until the algorithm finds a value $F\left(\xi_{i}\right)$ that is below the prescribed threshold, or if a maximum number of iterations is reached. Clearly, this approach may be computationally expensive in a general frame.

One possible way to reduce the computational cost required to minimize function $F$ is to use the set of estimates obtained at the end of the first iteration, viz. $F\left(\xi_{1}\right), \ldots, F\left(\xi_{N}\right)$, and approximate the whole function $F$ by using a surrogate model. The simplest choice to handle this problem is by fitting the set of data $F\left(\xi_{1}\right), \ldots, F\left(\xi_{N}\right)$ by a polynomial $P(\xi)$ at an order $M \leq N$. The mathematical problem then consists in finding the coefficients $\left\{p_{0}, p_{1}, . ., p_{M}\right\}$ of the polynomial $P(\xi)=p_{0}+p_{1} \cdot \xi+\ldots+p_{M} \cdot \xi^{M}$ that minimize the following sum of squares:

$$
\sum_{n=1}^{N}\left[P\left(\xi_{n}\right)-F\left(\xi_{n}\right)\right]^{2}
$$

We can notice that for large values of parameters $M$ (order of the polynomial approximation to function $F$ ) and $N$, as $M \leq N$, we can expect to approach $F$ by $P$ as closely as we want. Then, the polynomial approximation of $F$ can be used directly to provide an estimate $\xi(P)$, that depends on $P$, of $F(\xi)=0$.

The two step process that we have just described (sampling of the output of a given model using random inputs followed by a polynomial approximation of the "response" curve) is the main principle of Polynomial Chaos (PC) approaches (although PC can also be implemented in dimensions higher than 1 , viz. when more than one random variable is used to determine 
the input-output relationship, a problem that we will not consider here). More details about $\mathrm{PC}$ are given in the next section.

\section{THE POLYNOMIAL CHAOS (PC) APPROACH.}

The principle of Polynomial Chaos expansion was introduced by Wiener in 1938 [13], under the name "Homogeneous Chaos" (HC). HC mainly consists in expanding any stochastic field/process into an infinite series of orthogonal Hermite Polynomials of Gaussian random variables. Such a series expansion converges for any $L^{2}$ functional, that is to say stochastic processes with finite second moments, as a consequence of Cameron and Martin's theorem [15]. PC method was extended in Ref. [16] to a larger family of orthogonal polynomials (based on the so-called Askey scheme) and types (uniformly distributed over a bounded interval $[a, b]$ for instance) of random variables: the technique is then called generalized PC (gPC). Recently, PC approach was stretched to any possible kind of polynomials in Ref. [17]. This last method is referred to as the arbitrary PC (aPC) and uses directly the data to construct the basis of orthogonal polynomials that is the most suited to handle the calculation of the coefficients in the series. More details about those various PC techniques are summarized in Refs. [16-18]. Here, we will only describe the main ideas.

Let $\boldsymbol{\xi}=\left(\begin{array}{llll}\xi_{1} & \xi_{2} & \cdots & \xi_{m}\end{array}\right)$ be a vector of random inputs for some model $f=f(\boldsymbol{\xi})$. Notice that notations $\xi_{i}$ do not have the same meaning as in the previous section as they represent here components of a $m$-dimensional random vector. Previously, the same notation was used to signify $N$ samples of a random variable $\xi$ in dimension 1 .

$f$ may be defined explicitly, in closed form, or implicitly, in terms of a set of differential equations for instance. The principle of the PC framework is to approximate the response of the model by a truncated series expansion that takes the following form:

$$
f(\boldsymbol{\xi}) \approx \sum_{i=0}^{M} f_{i} \cdot \pi_{i}(\boldsymbol{\xi})
$$


where $\pi_{i}(\boldsymbol{\xi})$ is a product of orthogonal polynomials associated to each of the components of random vector $\boldsymbol{\xi}$. The total number of terms $M$ in expansion (5) depends on the dimension of vector $\boldsymbol{\xi}, m$, and on the order of the polynomial representation for each component, $p$. It is given by the following formula (from Ref. [16]):

$$
M+1=\frac{(m+p) !}{m ! p !}
$$

For one dimensional problems viz. $m=1$ as considered here, polynomials $\pi_{i}(\boldsymbol{\xi})$ reduce to usual orthogonal polynomials and obviously, from Eq. (6), $M=p$. From now on, we will restrict our talk to this case. Consequently, we consider Eq. (5) written in the following simpler form:

$$
f(\xi) \approx \sum_{i=0}^{M} f_{i} \cdot \pi_{i}(\xi)
$$

The main difficulty consists in finding the coefficients $f_{i}$ that appear in the previous expansion. This problem can be readily solved if: $1 / \xi$ is distributed according to a known continuous probability density $\Omega(\xi)$ and 2/ polynomials $\pi_{i}(\xi)$ are chosen orthogonal with respect to the corresponding probability weights $d \Omega(\xi)=\omega(\xi) \cdot d \xi$ (over the domain $\mathscr{D}$ over which the random variable $\xi$ takes values), viz.:

$$
\int_{D} \pi_{i}(\xi) \cdot \pi_{j}(\xi) \cdot \omega(\xi) d \xi\left\{\begin{array}{l}
=0 \text { if } i \neq j \\
=\left\langle\pi_{i} \mid \pi_{i}\right\rangle_{\omega} \neq 0 \text { if } i=j
\end{array}\right.
$$

In Eq. (8), $\langle\mid\rangle_{\omega}$ represents the inner product in the space of variable $\xi$.

In fact, in this case, the coefficients that appear in Eq. (7) can be obtained directly as:

$$
f_{i}=\frac{1}{\left\langle\pi_{i} \mid \pi_{i}\right\rangle_{\omega}} \cdot \int_{D} \pi_{i}(\xi) \cdot f(\xi) \cdot \omega(\xi) d \xi=\frac{\left\langle\pi_{i} \mid f\right\rangle_{\omega}}{\left\langle\pi_{i} \mid \pi_{i}\right\rangle_{\omega}}
$$


One usual approach (called non-intrusive, $[18,19]$ ) to handle the computation of coefficients given by Eq. (9) consists in using simulations of the response function of model $f$ to various random inputs $\xi$ and then use a projection (see Eq. (9)) of this response against each basis polynomial $\pi_{i}(\xi)$. This process can be handled very easily by a Monte Carlo method. Other techniques, usually called intrusive, exist. They are both discussed, for instance, in Ref. [18].

Fundamentally, the two methods described in the previous sections are the same, but formulated in a slightly different way. Indeed, they consist in the two steps: 1/ feed a model with some random variable of known probability density, 2/ approximate the output of the model by a polynomial. We will refer to them as Polynomial Chaos Expansions (PCE) in the following.

\section{APPLICATION OF PCE IN THE FRAME OF GAS RADIATION MODELING.}

In this section, we are going to show how it is possible to approximate any transmission function using a PCE.

We can reformulate the problem set in section 1 within the PC framework. Indeed, following Eq. (7), and according to the previous section, we seek an approximation of the function $F$ as defined by Eq. (3) as:

$$
F(\xi) \approx \sum_{i=0}^{M} F_{i} \cdot \pi_{i}(\xi)
$$

The only things that we have to show in order to justify this polynomial form is, following Cameron-Martin's theorem, that the "process" $\xi \rightarrow F(\xi)$ has a finite variance. In other words, that: 


$$
\left\{\begin{array}{l}
\left|\int_{0}^{1} F(\xi) d \xi\right|<+\infty \\
\int_{0}^{1}[F(\xi)]^{2} d \xi<+\infty
\end{array}\right.
$$

Those two conditions are obviously correct here since (from Eq. (3-a)):

$$
\text { for any } \xi \in[0,1],-1 \leq F(\xi) \leq 1
$$

Following section 1 , variable $\xi$ is randomly chosen in the interval $[0,1]$. Then, Table 4.1 from Ref. [16] suggests to use the shifted-Legendre polynomials $P_{i}^{*}(\xi)$ (using the notations used in Ref. [20]) for $\pi_{i}(\xi)$. The coefficients of the PCE of the transmission function defined by Eq. (10) are simply, in this case:

$$
F_{i}=\int_{0}^{1} P_{i}^{*}(\xi) \cdot F(\xi) d \xi
$$

Quantities $F_{i}$ are the coefficients of the shifted Legendre series expansion of function $F$. They depend on the two thermophysical states $\underline{\phi}^{\text {ref }}$ and $\underline{\phi}$, and the approach described in Refs. $[9,10]$ can be used to reformulate the previous equations. Indeed, Eq. (13) can be rewritten in terms of CDPs (see Appendix). This change of polynomial basis transforms the set of equations Eqs. $(10,13)$ into:

$$
F(\xi)=F(\xi=0)+\sum_{i=0}^{M-1} \beta_{i} \cdot \Phi_{i}^{(M)}(\xi)
$$

where $\Phi_{i}^{(M)}$ are the Cutteridge-Devyatov polynomials at order $M$ and:

$$
\beta_{i}=\beta_{i}\left(\underline{\phi}^{r e f}\right)=\int_{0}^{1} \xi^{i} \cdot \frac{d F(\xi)}{d \xi} d \xi=\int_{0}^{1} \xi^{i} \cdot \frac{d \tau^{\Delta \eta}\left[L(\xi), \underline{\phi}^{r e f}\right]}{d \xi} d \xi
$$

are generalized moments. In Eq. (14-a), the term $F(\xi=0)$ appears as a consequence of the usual definition of CDPs (see Eq. (A.3)). The same definition requires the summation in the second term at the RHS in Eq. (14-a) to extend from index $i=0$ to $i=M-1$. 
Indeed, following the definition of $F$ (see Eq. (3-a)), it is obvious that:

$$
\frac{d F(\xi)}{d \xi}=\frac{d \tau^{\Delta \eta}\left[L(\xi), \underline{\phi}^{r e f}\right]}{d \xi}
$$

Usually, the main difficulty in PC is to estimate the coefficients $\beta_{i}\left(\underline{\phi}^{\text {ref }}\right)$. They can be calculated by application of a Monte Carlo method, as in usual PC approaches. But in our case, they can also be approximated directly from LBL data as they are simply (from Eq. (14b)):

$$
\beta_{i}=\beta_{i}\left(\underline{\phi}^{r e f}\right)=\lim _{J \rightarrow+\infty} \sum_{j=1}^{J-1} \xi_{j}^{i}\left\{\tau^{\Delta \eta}\left[L\left(\xi_{j+1}\right), \underline{\phi}^{r e f}\right]-\tau^{\Delta \eta}\left[L\left(\xi_{j}\right), \underline{\phi}^{r e f}\right]\right\}
$$

where $\xi_{j}=\frac{j-1}{J-1}$. It should be noticed that the calculation of the generalized moments in this very simple way is due to the rewriting of the Legendre polynomials in terms of CDPs. Application of Legendre polynomials is obviously possible (see Eq. (13)) but is clearly less convenient.

Now, as soon as coefficients $\beta_{i}$ are known, it is possible to rewrite Eq. (1) as:

$$
F(\xi=0)+\sum_{i=0}^{M-1} \beta_{i} \cdot \Phi_{i}^{(M)}(\xi)=0
$$

Applying PCE thus enables to transform a highly non-linear equation, Eq. (1), into a simpler polynomial one. Indeed, the solution $u$ to Eq. (1) can be deduced from the solution $\xi_{u}$ to Eq. (17) by a direct application of Eq. (2): $u=u_{\min } \cdot\left(u_{\max } / u_{\min }\right)^{\xi_{u}}$.

But this formulation also has interesting applications. In fact, let us choose arbitrarily $L^{r e f}=1$ cm and define the interval $\left[u_{\min }, u_{\max }\right]$ so that:

$$
\left\{\begin{array}{l}
\tau^{\Delta \eta}\left(L_{\min }=u_{\min } \cdot L^{r e f}, \underline{\phi}^{r e f}\right)=1-\varepsilon \\
\tau^{\Delta \eta}\left(L_{\max }=u_{\max } \cdot L^{r e f}, \underline{\phi}^{r e f}\right)=\varepsilon^{\prime}
\end{array}\right.
$$


where $0<\varepsilon \ll 1$ and $0<\varepsilon^{\prime} \ll 1$ are two real numbers. Obviously, from Eq. (18), the values of $L_{\min }$ and $L_{\max }$ depend on $\underline{\phi}^{\text {ref }}$.

Then, for any path length $L$ inside the interval $\left[L_{\min }=L^{r e f} \cdot u_{\min }, L_{\max }=L^{r e f} \cdot u_{\max }\right]$, the application of Eq. (14-a) provides, with $\xi=\ln \left(L / L_{\min }\right) / \ln \left(L_{\max } / L_{\min }\right)$ :

$$
F(\xi)=\tau^{\Delta \eta}\left[L(\xi), \underline{\phi}^{r e f}\right]-\tau^{\Delta \eta}\left(L^{r e f}, \underline{\phi}\right)=\tau^{\Delta \eta}\left[L(\xi=0), \underline{\phi}^{r e f}\right]-\tau^{\Delta \eta}\left(L^{r e f}, \underline{\phi}\right)+\sum_{i=0}^{M-1} \beta_{i}\left(\underline{\phi}^{r e f}\right) \cdot \Phi_{i}^{(M)}(\xi)
$$

that shows that the transmission function in the thermophysical state $\underline{\phi}^{\text {ref }}$ can be estimated, over the range $\left[L_{\min }=L^{r e f} \cdot u_{\min }, L_{\max }=L^{r e f} \cdot u_{\max }\right]$ of values of $L$, and as a direct consequence of Eq. (19), as:

$$
\tau^{\Delta \eta}\left(L, \underline{\phi}^{r e f}\right)=\tau^{\Delta \eta}\left(L_{\min }, \underline{\phi}^{r e f}\right)+\sum_{i=0}^{M-1} \beta_{i}\left(\underline{\phi}^{r e f}\right) \cdot \Phi_{i}^{(M)}\left[\xi=\ln \left(L / L_{\min }\right) / \ln \left(L_{\max } / L_{\min }\right)\right]
$$

In other words, Eq. (20) can be used to estimate transmission spectra in the reference state $\underline{\phi}^{r e f}$ (that can be chosen arbitrarily, which means that the previous formula can be applied for any state $\underline{\phi}$ by calculating the appropriate sets of coefficients $\left.\beta_{i}(\underline{\phi})\right)$ over the range of lengths $\left[L_{\min }=L_{\min }(\underline{\phi})=L^{r e f} \cdot u_{\min }(\underline{\phi}), L_{\max }=L_{\max }(\underline{\phi})=L^{r e f} \cdot u_{\max }(\underline{\phi})\right]$. By choosing "infinitely small" values for parameters $\varepsilon$ and $\varepsilon^{\prime}$, the set of equations Eq. (20), for the calculation of transmissivities, together with Eq. (17) to handle non-uniformities (assuming that gas spectra are scaled), thus enables to propose a very simple polynomial model for transmission spectra. Its accuracy is assessed against LBL reference calculations in uniform and non-uniform situations in the next section. 


\section{APPLICATION OF THE CUTTERIDGE-DEVYATOV-POLYNOMIAL CHAOS APPROXIMATION OF TRANSMISSION SPECTRA IN UNIFORM AND NON- UNIFORM MEDIA.}

\subsection{LBL data and model parameters.}

In the present work, we consider $\mathrm{CO}_{2}-\mathrm{N}_{2}$ mixtures only. The LBL code used for the building of the approximate model was described in Ref. [7]. The interested reader should refer to this paper for additional details. Calculations are based on the CDSD-4000 spectroscopic database [21]. The C- $k$ model used in the present work was built following the same method as described in Ref. [8].

The LBL database contains gas spectra at high resolution $\left(10^{-2} \mathrm{~cm}^{-1}\right)$ calculated for temperatures between $300 \mathrm{~K}$ and $5000 \mathrm{~K}$ with a $100 \mathrm{~K}$ step at $0.1 \mathrm{CO}_{2}$ molar fraction. All calculations are at atmospheric pressure.

The building of the model parameters requires two steps:

- the first one is to find values for parameters $L_{\min }=L_{\min }(\underline{\phi})$ and $L_{\max }=L_{\max }(\underline{\phi})$ for any thermophysical state, $\underline{\phi}$. In order to estimate those quantities, two methods were considered: Method M1 - values of $L_{\min }$ and $L_{\max }$ are fixed to $5 \cdot 10^{-1} \mathrm{~cm}$ and $50 \mathrm{~cm}$ respectively (those choices were made to ensure that the model would be able to handle the calculations described in the next sections, both in uniform and nonuniform situations); Method M2 - the interval $\left[10^{-6}, 10^{6}\right] \mathrm{cm}$ is discretized into 1000 logarithmically scaled values and the band averaged transmission function is calculated at each of these points. This provides an estimate of the so-called Curve-OfGrowth (COG) that represents the transmissivity as a function of the path in the gas. Then, the choice $\varepsilon=\varepsilon^{\prime}=10^{-4}$ is done and solutions $L_{\min }$ and $L_{\max }$ to Eqs. (18) are 
estimated directly by interpolating (linearly with respect to $\xi$ ) between the two closest values (of both $1-\varepsilon$ and $\varepsilon^{\prime}$ ) found along the COG.

- the second step consists in evaluating the generalized moments using Eq. (16). This calculation was done for all the thermophysical conditions, and with a value for parameter $J$ (see Eq. (16)) equal to 5000 (chosen arbitrarily). Moments up to $M+1=14$ were calculated this way.

The total size of the database is about 6 Mbytes.

In order to keep the model as simple as possible, for $\mathrm{CO}_{2}$ (for which only an interpolation of the model with respect to temperature is required), we propose to calculate transmission spectra for thermophysical states not included in the dataset by using a simple linear interpolation scheme:

$$
T_{i} \leq T \leq T_{i+1} \Rightarrow \tau^{\Delta \eta}(L, T) \approx \frac{T_{i+1}-T}{T_{i+1}-T_{i}} \cdot \tau^{\Delta \eta}\left(L, T_{i}\right)+\frac{T-T_{i}}{T_{i+1}-T_{i}} \cdot \tau^{\Delta \eta}\left(L, T_{i+1}\right)
$$

This simple approach is assessed against LBL calculation in the next section.

\subsection{Application in uniform cases.}

Two of the three test cases considered here are taken from Ref. [22]. They correspond to 10 $\mathrm{cm}$ of mixtures of $10 \% \mathrm{CO}_{2}$ and $90 \%$ of $\mathrm{N}_{2}$ at $300 \mathrm{~K}$ (Figure 1), $1500 \mathrm{~K}$ (Figure 2) and 2900 K (Figures 3). They do not involve the interpolation scheme described in the previous section. Each time, results for the model with parameters obtained from Method M1 (with 10 moments) and M2 (with 14 moments) are given. They are also compared to calculations made with a usual C- $k$ model (as detailed earlier in this section).

The order of magnitude of the absolute difference between LBL reference calculations and the CDPC polynomial approximations (for both methods, viz. M1 with 10 moments and M2 with 14 moments) is lower than $5 \cdot 10^{-3}$, for all cases. Method M1 with 10 moments is more accurate than M2 with 14 moments, and it provides an accuracy that is almost the same as usual C- $k$ models. 
Figure 4 depicts results for a case with interpolation. The temperature of the gas was arbitrarily chosen as $2950 \mathrm{~K}$ (which is outside our dataset). Again, a $\mathrm{CO}_{2} / \mathrm{N}_{2}$ mixture is considered $\left(\mathrm{CO}_{2}\right.$ molar fraction is 0.2$)$. The same path length as used previously $(10 \mathrm{~cm})$ was considered. The error is found to be of the same order of magnitude as in the uniform cases without interpolation, for both CDPC approaches.

\subsection{Application in non-uniform cases.}

The last test case was proposed in Ref. [3]. It consists of a non-uniform gas path divided in two parts: a hot one, at $T_{h}=1000 \mathrm{~K}$, and a cold one, at $T_{c}=300 \mathrm{~K}$. The lengths of the layers are $L_{h}=L_{c}=50 \mathrm{~cm}$. They contain a mixture of $10 \% \mathrm{CO}_{2}$ and $90 \% \mathrm{~N}_{2}$. The $\mathrm{CO}_{2}$ molar fraction is thus 0.1 in both columns.

The steps to calculate the transmission function of the non-uniform column are the following ones:

1/ choose a reference state. Here we have arbitrarily chosen the hot column as the reference viz. $\underline{\phi}^{r e f}=\underline{\phi}_{h}$.

2/ estimate the transmission function of the cold column using Eq. (20):

$$
\tau^{\Delta \eta}\left(L_{c}, \underline{\phi}_{c}\right)=\tau^{\Delta \eta}\left(L_{\min }, \underline{\phi}_{c}\right)+\sum_{i=0}^{M-1} \beta_{i}\left(\underline{\phi}_{c}\right) \cdot \Phi_{i}^{(M)}\left[\xi=\ln \left(L_{c} / L_{\min }\right) / \ln \left(L_{\max } / L_{\min }\right)\right]
$$

In the previous equation, $L_{\min }$ and $L_{\max }$ correspond to the solutions of Eqs. (18) in which we have replaced $\underline{\phi}^{\text {ref }}$ by $\underline{\phi}_{c}$.

3/ evaluate the solution to (from Eq. (17) - This polynomial equation was solved, in this work, by application of the bisection method. $L_{\min }$ corresponds here to the solution of Eq. (18) in which we have replaced $\underline{\phi}^{\text {ref }}$ by $\underline{\phi}_{h}$ ):

$$
\tau^{\Delta \eta}\left(L_{c}, \underline{\phi}_{c}\right)=\tau^{\Delta \eta}\left[L\left(\xi_{c}\right)=u_{c} \cdot L_{c}, \underline{\phi}_{h}\right]=\tau^{\Delta \eta}\left(L_{m i n}, \underline{\phi}_{h}\right)+\sum_{i=0}^{M-1} \beta_{i}\left(\underline{\phi}_{h}\right) \cdot \Phi_{i}^{(M)}\left(\xi_{c}\right)
$$

and estimate the corresponding scaling coefficient: 


$$
u_{c}=\frac{L\left(\xi_{c}\right)}{L_{c}}=\frac{L_{\min }\left(\underline{\phi}_{h}\right)}{L_{c}}\left[\frac{L_{\max }\left(\underline{\phi}_{h}\right)}{L_{\min }\left(\underline{\phi_{h}}\right)}\right]^{\xi_{c}}
$$

4/ finally, calculate the transmissivity of the non-uniform path $\tau^{\Delta \eta}$ (non-uniform path) as:

$$
\begin{aligned}
\tau^{\Delta \eta}(\text { non-uniform path }) & =\tau^{\Delta \eta}\left(L=u_{c} L_{c}+L_{h}, \underline{\phi}^{\text {ref }}=\underline{\phi}_{h}\right) \\
& =\tau^{\Delta \eta}\left(L_{\min }, \underline{\phi}_{h}\right)+\sum_{i=0}^{M-1} \beta_{i}\left(\underline{\phi}_{h}\right) \cdot \Phi_{i}^{(M)}\left[\xi=\ln \left(L / L_{\min }\right) / \ln \left(L_{\max } / L_{\min }\right)\right]
\end{aligned}
$$

In Eq. (25), $L_{\min }$ and $L_{\max }$ correspond to the solutions of Eqs. (18) in which we have replaced $\underline{\phi}^{\text {ref }}$ by $\underline{\phi}_{h}$.

The flowchart that corresponds to this calculation is given in Figure 5. Results are depicted in Figure 6. Again, we can observe that the model is very accurate, as the two CDPC approaches provide results that have the same accuracy as usual $\mathrm{C}-k$ models. Errors are nevertheless higher than in uniform cases but, in this situation, the two CDPC models are found to yield almost the same accuracy. This simply means that errors are mainly due to the application of the scaling assumption used to handle path non-uniformities.

\subsection{Discussion.}

The two CDPC approximations considered in the previous section are able to provide results that are close to usual C- $k$ models, both in uniform and non-uniform media. The use of 14 moments requires calculations in quadruple precision (this point was already emphasized in Ref. [10]) and thus is more time consuming than the method with 10 moments. Approach M2 with 14 moments is less accurate that method M1 with 10 moments. This can be easily understood as method M2 usually provides wider ranges of path lengths than M1, and adding more moments is not sufficient to maintain a similar accuracy between the two methods. But by using M1 with 10 moments and "small" intervals of path lengths (they however extend over 2 orders of magnitude), the approximate model is found to achieve LBL accuracy. 
One possible way to combine the advantages of the two approaches would be: $1 /$ to use method M2, in order to find the full range of applicability of the technique and, $2 /$ to split the resulting domain into smaller intervals, over which low order polynomials approximations could be used. This technique will be studied in future works.

For applications in non-uniform media, both methods provide the same accuracy: this is because the main source of errors is associated to the use of the scaling assumption of gas spectra to handle non-uniformities. Using this assumption, instead of correlation, was found to provide similar results in Ref. [3]. The present work confirms this result.

In terms of computational cost, some tests were made to compare the following models: CDPC with 10 generalized moments, C- $k$ with 7 and 15 values of the absorption coefficient (results provided for the C- $k$ model in the previous sections used 15 values of $k$ ) and SNB with the Malkmus' distribution of linestrengths. All calculations were performed in double precision. Narrow band transmissivities were calculated for the same band of $\mathrm{CO}_{2}$ (centered at $2400 \mathrm{~cm}^{-1}$ ) for $323 \cdot 10^{6}$ values of path lengths logarithmically scaled between $0.5 \mathrm{~cm}$ and 50 $\mathrm{cm}$ (computational times are thus representative of the cost of a given model to evaluate $10^{6}$ spectra of uniform columns of $\mathrm{CO}_{2}$ over 323 narrow bands). For all models, parameters (absorption coefficients for C- $k$, generalized moments for CDPC, etc) were pre-calculated and the associated computational times were not included in the comparison. For CDPC, Horner's method was used. This required rewriting Eq. (20) under the same form as in Eq. (A.8) (see Appendix). The computational time was found to be 34 seconds for the SNB model, 57 seconds for the CDPC approach, 68 and 131 seconds for the C- $k$ models with respectively 7 and 15 values of $k$ on one core of an Intel XEON CPU E5620 @ $2.40 \mathrm{~Hz}$. The C- $k$ model (both with 7 and 15 absorption coefficients) is thus more computationally demanding than the CDPC model with 10 generalized moments (that is itself almost $40 \%$ more time consuming than the SNB model). This result shows that the CDPC approach, which was shown in the 
previous sections to be very accurate, is also promising in terms of computational resources. Nevertheless, further work is required to confirm this result for applications in non-uniform media.

\section{CONCLUSION.}

A simple and accurate approximate model has been proposed for the narrow band transmissivities of gases in uniform and non-uniform media. The approach is based on some results from Polynomial Chaos and Generalized Moment methods. The technique has been applied for the calculation of transmission spectra of $\mathrm{CO}_{2}-\mathrm{N}_{2}$ mixtures at 3 temperatures: $300 \mathrm{~K}, 1500 \mathrm{~K}$ and $2900 \mathrm{~K}$. Results show that the method is reliable in uniform situations. Furthermore, comparison of the proposed approach with results obtained by application of a usual C- $k$ model demonstrates that the present model is reliable, both in uniform and nonuniform situations. Its coupling with the Multi-Spectral (MS) approach is natural, as MS describes methods to create spectral interval over which gas spectra can be reasonably treated as scaled [7,8], as assumed here to handle non-uniformities. The use of the CDPC approximation within the Multi-Spectral framework will be studied in future works. 


\section{REFERENCES}

[1] MODEST MF. Radiative heat transfer, $2^{\text {nd }}$ ed. New York: Academic Press; 2003 (ISBN 012-503163-7).

[2] TAINE J, SOUFIANI A. Gas IR radiative properties: From spectroscopic data to approximate models, Adv. Heat Transfer 1999;33:295-414.

[3] MODEST MF. Narrow-band and full-spectrum $k$-distributions for radiative heat transfer correlated- $k$ vs. scaling approximation, JQSRT 2003;76:69-83.

[4] ZHANG H, MODEST MF. Multi-group full-spectrum $k$-distribution database for water vapor mixtures in radiative transfer calculations, IJHMT 2003;46:3593-3603.

[5] ZHANG H, MODEST MF. Scalable multi-group full-spectrum correlated- $k$ distributions for radiative transfer calculations, JHT 2003;125:454-461.

[6] LEVI DI LEON R, TAINE J. A fictive gas-method for accurate computations of lowresolution IR gas transmissivities: application to the $4.3 \mu \mathrm{m} \mathrm{CO}_{2}$ band, Rev. Phys. Appl. $1986 ; 21: 825-831$.

[7] ANDRE F, HOU L, ROGER M, VAILLON R. The multispectral gas radiation modeling: a new theoretical framework based on a multidimensional approach to $k$ distribution methods, JQSRT 2014;147:178-195.

[8] ANDRE F, HOU L, SOLOVJOV V.P. An exact formulation of $k$-distribution methods in non-uniform gaseous media and its approximate treatment within the Multi-Spectral framework, in: Proceeding of Eurotherm Seminar 2015 (CTRPM5), Albi, France.

[9] ANDRE F, SOLOVJOV V.P., VAILLON R, LEMONNIER D. Modeling the cumulative distribution of absorption coefficients of gases using the Generalized $k$-moment method, JQSRT 2013;124:49-61. 
[10] ANDRE F, SOLOVJOV V.P., HOU L, VAILLON R. The Generalized $k$-moment method for the modeling of cumulative $k$-distributions of $\mathrm{H}_{2} \mathrm{O}$ at high temperature, JQSRT 2014;143:92-99.

[11] DEVYATOV B.N., RUBTSOV N.A., SOLOVJOV V.P., CHIRCASHENKO E.P. General method of solving complex heat and radiation transfer problems, Russian Journal of Engineering Thermophysics 1992;2:167-191.

[12] DEVYATOV B.N., RUBTSOV N.A., SOLOVJOV V.P. General method for analytic study of nonlinear time-varying problems of heat transfer and radiation transport, Sov. Phys. Dokl. 1991;36:846-849.

[13] WIENER N. The Homogeneous Chaos, American Journal of Mathematics 1938;60:897936.

[14] SCHNEIDER J.J., KIRKPATRICK S. Stochastic Optimization, Springer; 2006 (ISBN10 3-540-34559-0).

[15] CAMERON R.H., MARTIN W.T. The orthogonal development of non-linear functionals in series of Fourier-Hermite functionals, Annals of Mathematics, Second series, 1947;48:385392.

[16] XIU D, KARNIADAKIS G.M. The Wiener-Askey polynomial chaos for stochastic differential equations, SIAM J. Sci. Comput. 2002;24:619-644.

[17] OLADYSHKIN Q., NOWAK W. Data-driven uncertainty quantification using the arbitrary polynomial chaos expansion, Reliability Engineering and System Safety 2012;106:179-190.

[18] PER PETTERSSON M., IACCARINO G., NORDSTROM J. Polynomial chaos methods for hyperbolic partial differential equations - Numerical techniques for fluid dynamics problems in the presence of uncertainties, Springer; 2015 (ISBN 978-3-319-10713-4). 
[19] ELBRED M.S., WEBSTER C.G., CONSTANTINE P.G. Evaluation of non-intrusive approaches for Wiener-Askey generalized polynomial chaos, in: Proceeding of the $49^{\text {th }}$ AIAA/ASME/ASCE/AHS/ASC Structures, Structural Dynamics and Materials Conference, 7-10 April 2008, Schaumburg, IL.

[20] ABRAMOWITZ M., STEGUN I.A., Handbook of Mathematical Functions with formulas, Graphs, and Mathematical Tables, New York: Dover 1965.

[21] TASHKUN, S.A., PEREVALOV, V.I., CDSD-4000: High resolution, high temperature carbon dioxide spectroscopic databank, JQSRT 2011;112:1403-1410.

[22] RIVIERE Ph., SOUFIANI A. Updated band model parameters for $\mathrm{H}_{2} \mathrm{O}, \mathrm{CO}_{2}, \mathrm{CH}_{4}$ and CO radiation at high temperature, IJHMT 2012;55:3349-3358.

[23] CHIHARA T.S. An introduction to orthogonal polynomials, Dover; 1978 (ISBN-10: 0486-47929-3) 
FIGURE CAPTIONS

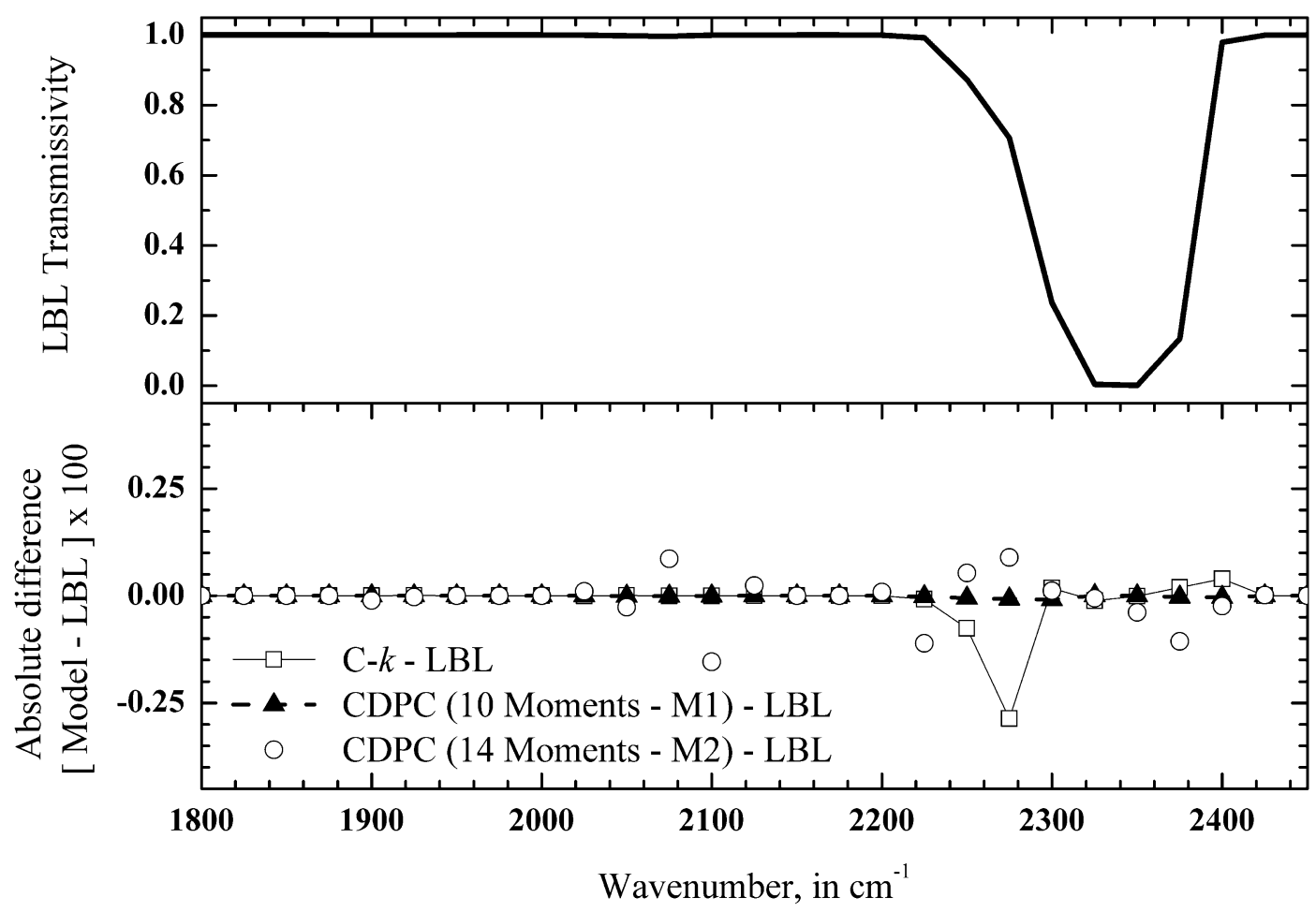

Figure 1. Narrow band $\left(25 \mathrm{~cm}^{-1}\right)$ transmission function for $10 \mathrm{~cm}$ of $10 \% \mathrm{CO}_{2}-90 \% \mathrm{~N}_{2}$ at $300 \mathrm{~K}$ and 1 atmosphere - Top: LBL reference calculation. Bottom: absolute difference $\mathrm{x} 10^{2}$ between LBL and approximate models (C- $k$, CDPC with methods M1 and M2). 


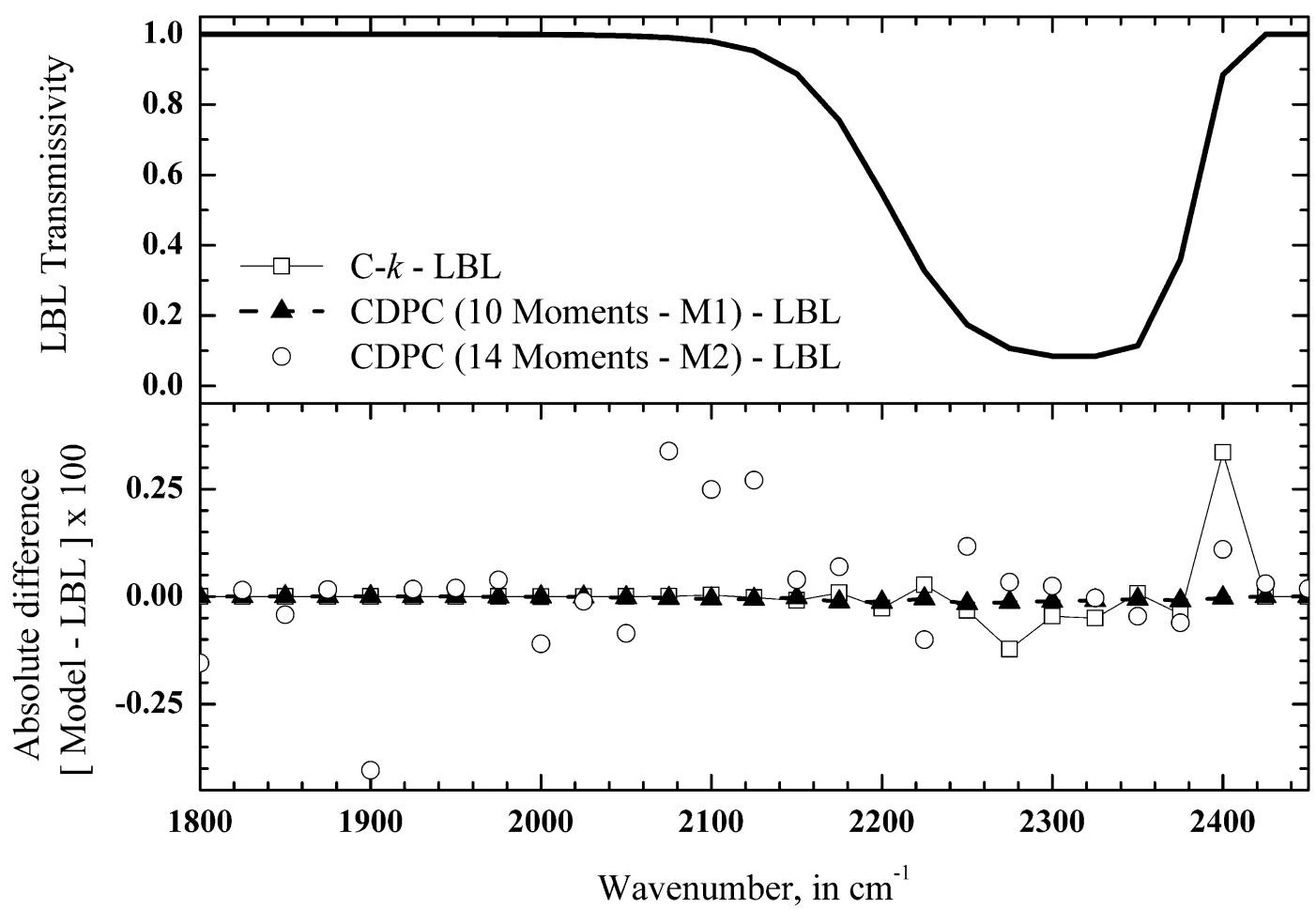

Figure 2. Narrow band $\left(25 \mathrm{~cm}^{-1}\right)$ transmission function for $10 \mathrm{~cm}$ of $10 \% \mathrm{CO}_{2}-90 \% \mathrm{~N}_{2}$ at $1500 \mathrm{~K}$ and 1 atmosphere - Top: LBL reference calculation. Bottom: absolute difference $\mathrm{x}$ $10^{2}$ between LBL and approximate models (C- $k$, CDPC with methods M1 and M2). 


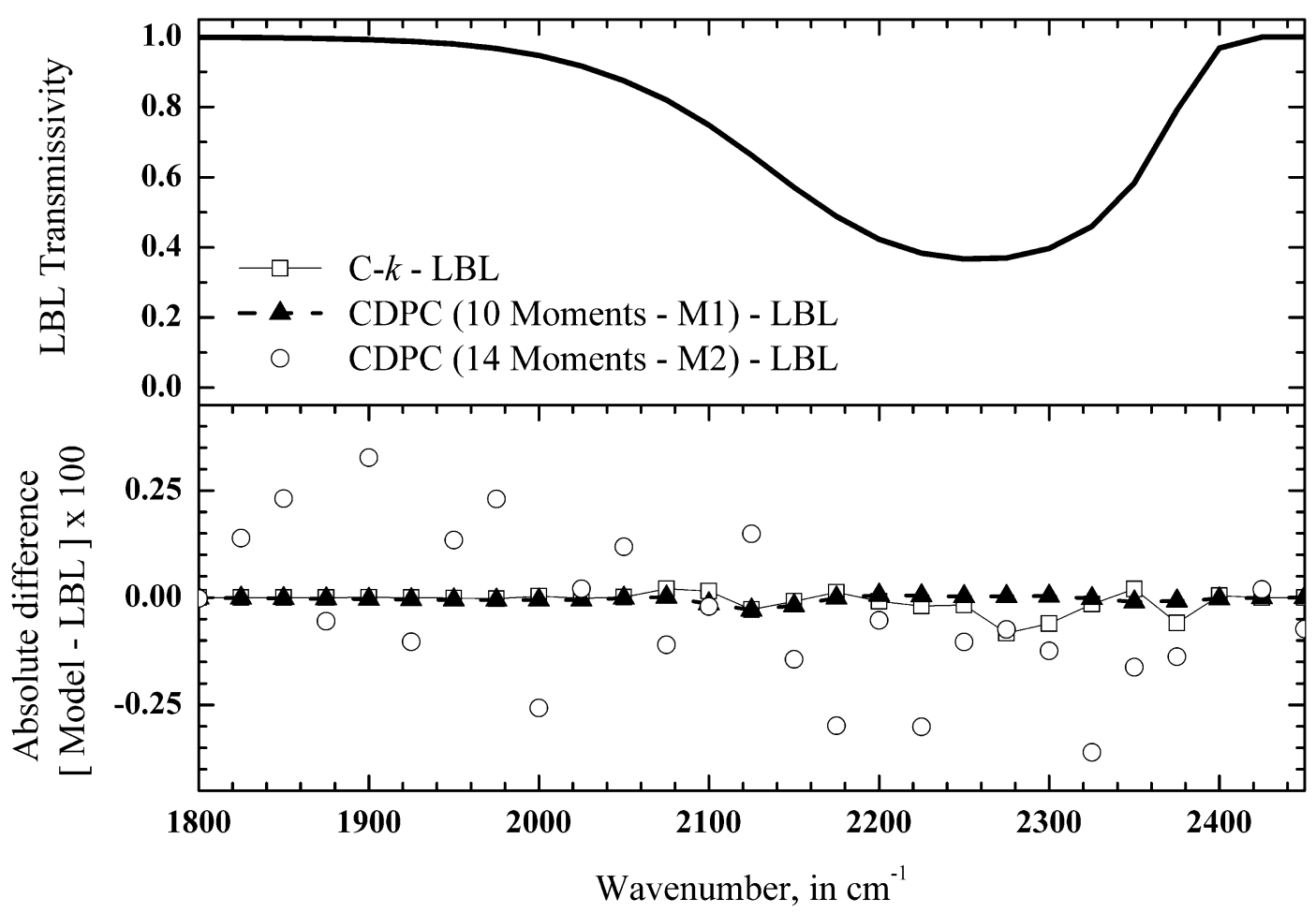

Figure 3. Narrow band $\left(25 \mathrm{~cm}^{-1}\right)$ transmission function for $10 \mathrm{~cm}$ of $10 \% \mathrm{CO}_{2}-90 \% \mathrm{~N}_{2}$ at $2900 \mathrm{~K}$ and 1 atmosphere - Top: LBL reference calculation. Bottom: absolute difference $\mathrm{x}$ $10^{2}$ between LBL and approximate models (C- $k$, CDPC with methods M1 and M2). 


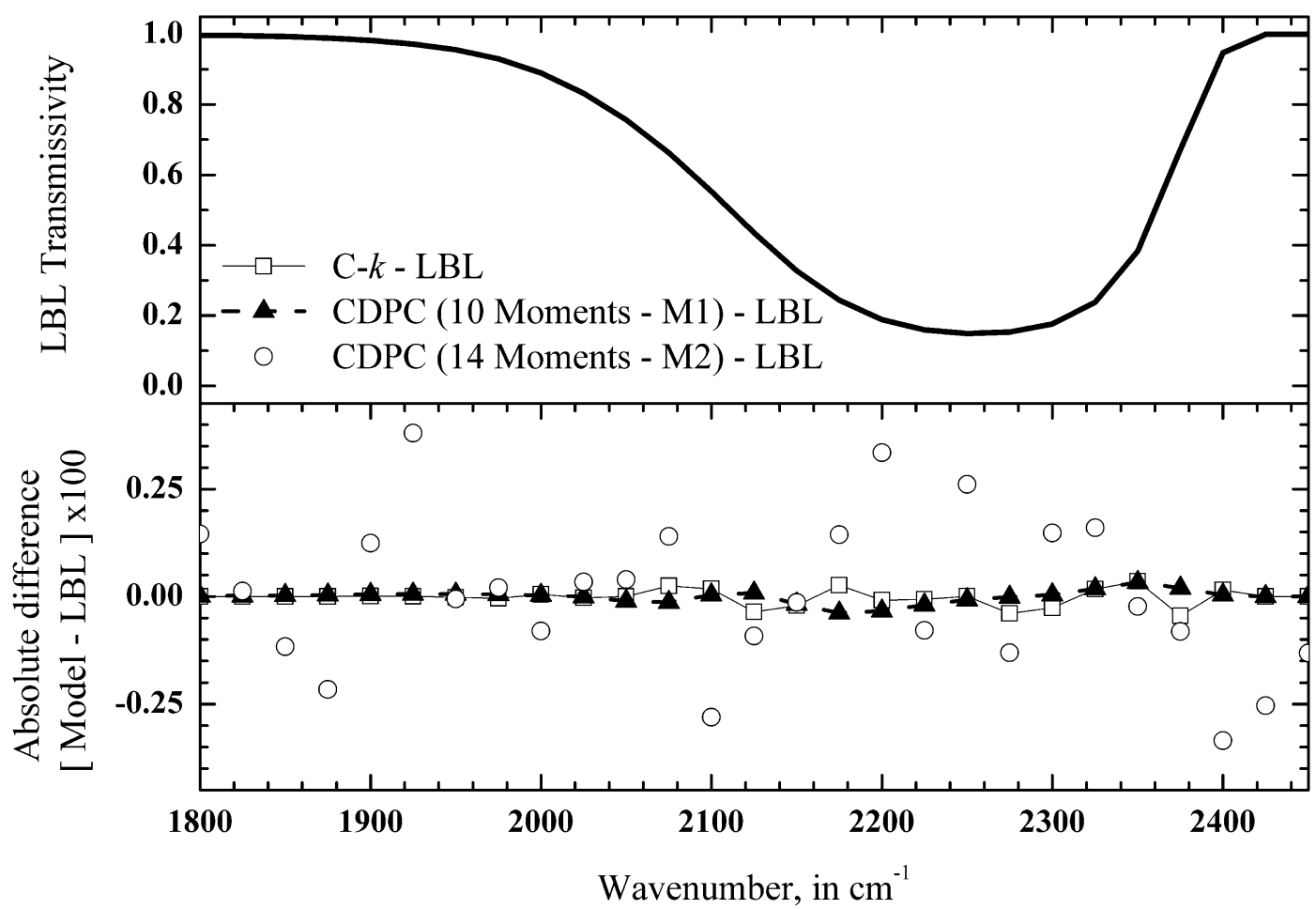

Figure 4. Narrow band $\left(25 \mathrm{~cm}^{-1}\right)$ transmission function for $10 \mathrm{~cm}$ of $20 \% \mathrm{CO}_{2}-80 \% \mathrm{~N}_{2}$ at $2950 \mathrm{~K}$ and 1 atmosphere - This calculation uses the interpolation scheme given by Eq .(21)

- Top: LBL reference calculation. Bottom: absolute difference x $10^{2}$ between LBL and approximate models (C-k, CDPC with methods M1 and M2). 


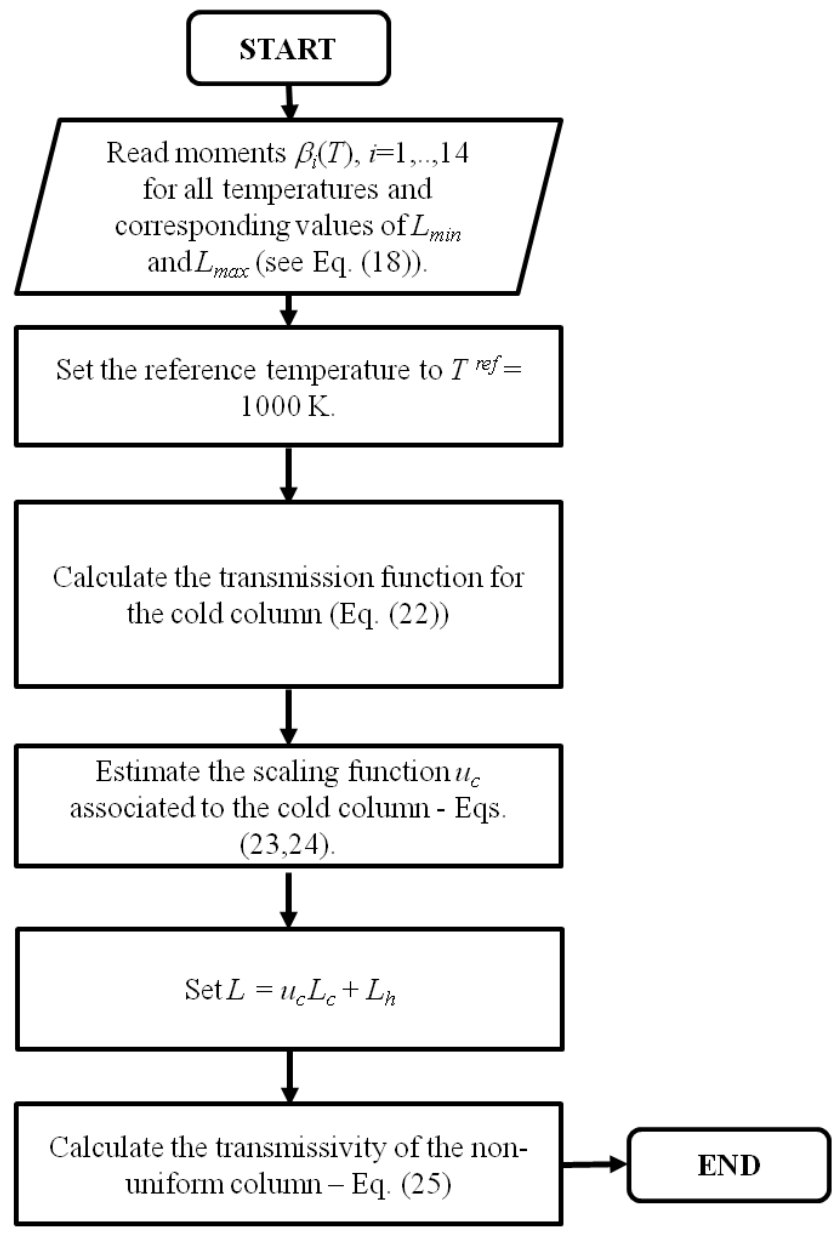

Figure 5. Flowchart for the application of the CDPC approximate model in non-uniform media (see text for details). 


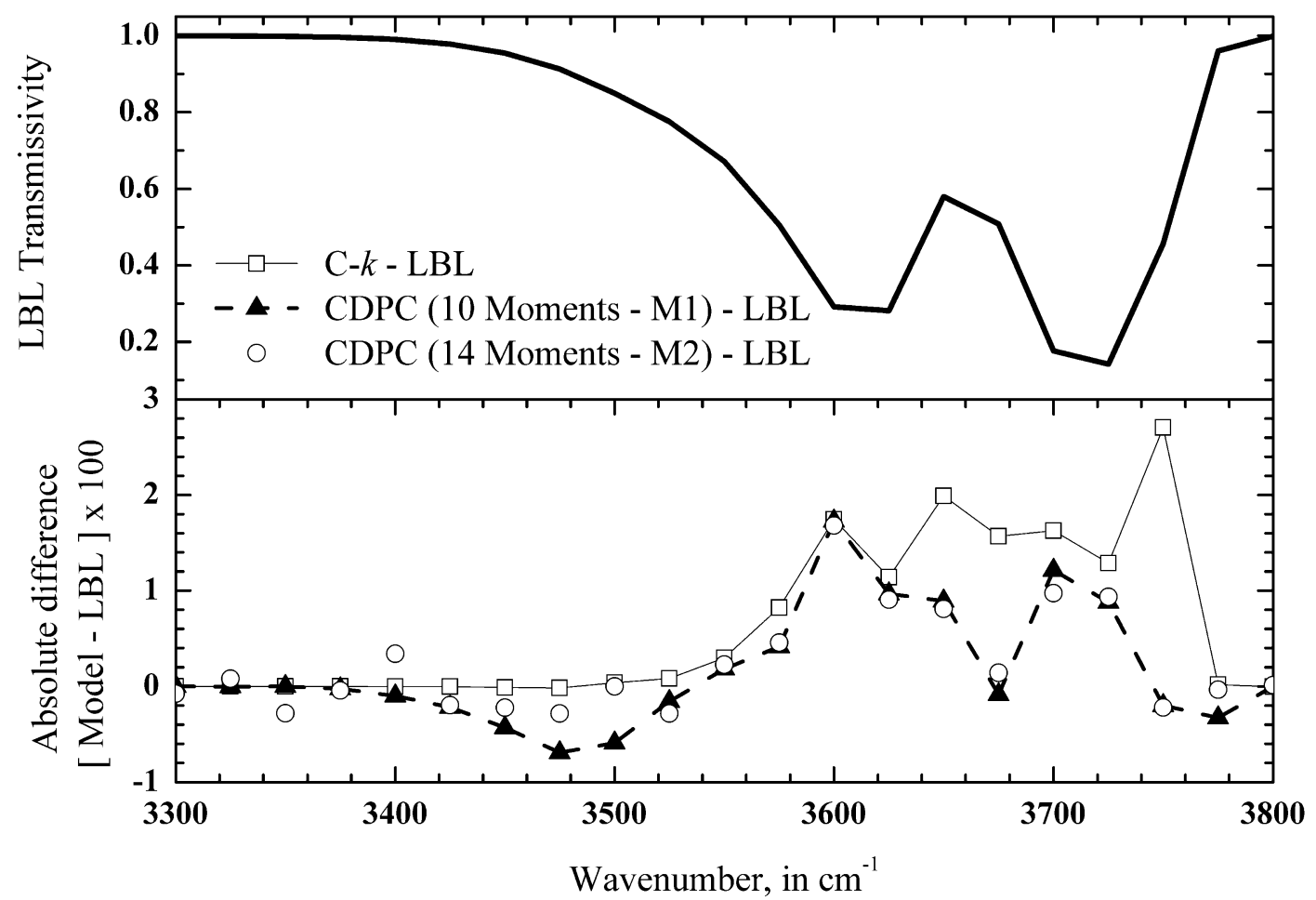

Figure 6. Narrow band $\left(25 \mathrm{~cm}^{-1}\right)$ transmission function for a non-uniform path (see text for details) - Top: LBL reference calculation. Bottom: absolute difference x $10^{2}$ between LBL and approximate models (C-k, CDPC with methods M1 and M2). 


\section{APPENDIX. THE CUTTERIDGE-DEVYATOV POLYNOMIAL CHAOS METHOD.}

The objective of this Appendix is to show that Devyatov's method of moments and Polynomial Chaos are two formulations of the same mathematical idea, but introduced in a different way and for different communities. This allows defining what can be called the "Cutteridge-Devyatov Polynomial Chaos" method.

\section{A.1. THE GENERALIZED $K$-MOMENT APPROACH AND CUTTERIDGE- DEVYATOV POLYNOMIALS (CDPs).}

The Generalized $k$-moment approach was proposed in Refs. $[9,10]$ as a tool to estimate cumulated $k$-distributions $g(k)$ of uniform gases. Following this method, function $g(k)$ can be approximated as a polynomial in the variable $\xi=\xi(k)=\ln \left(k / k_{\min }\right) / \ln \left(k_{\max } / k_{\min }\right)$ :

$$
g(k) \approx \sum_{p=0}^{N-1} l_{p} \cdot \Phi_{p}^{(N)}[\xi(k)]
$$

where the Generalized Moments $l_{p}, p=0, . ., N-1$ are given as:

$$
l_{p}=\frac{1}{\Delta \eta} \cdot \int_{\Delta \eta} \xi_{\eta}^{p} d \eta
$$

In Eq. (A.1), $\Phi_{p}^{(N)}[\xi(k)]$ are the Cutteridge-Devyatov Polynomials (CDPs) that can be calculated from:

$$
\Phi_{p}^{(N)}(\xi)=\sum_{j=0}^{N-1} \phi_{p j}^{(N)} \xi^{j+1}
$$

with the coefficients:

$$
\phi_{p j}^{(N)}=(-1)^{p+j} \frac{(N+p) !(N+j) !}{p !(p+1) ! j !(j+1) !(N-p-1) !(N-j-1) !} \frac{p+1}{p+j+1}
$$


More details about CDPs and how they are related to the inverse of the Hilbert matrix can be found in Ref. [10]. Those polynomials have the following property (called quasi-orthogonality in Ref. [23]):

$$
\text { for all } p \in\{0, . ., N-1\} \text { and } q \in\{0, . ., N-1\}, \int_{0}^{1} \xi^{q} \cdot \frac{d \Phi_{p}^{(N)}(\xi)}{d \xi} d \xi=\delta_{q p}
$$

where $\delta_{q p}$ is the usual Kronecker delta symbol which is 1 if $p=q$ and 0 if $p \neq q$.

In Eq. (A.2), $\xi_{\eta}$ represents the spectral value of the variable $\xi$, that is to say $\xi_{\eta}=\ln \left(\kappa_{\eta} / k_{\min }\right) / \ln \left(k_{\max } / k_{\min }\right)$. Obviously, the previous definition assumes that over the spectral interval $\Delta \eta$, all the values of the absorption coefficient fall inside a bounded interval $\left[k_{\min }, k_{\max }\right]$ with $k_{\min }>0$ (which means that no transparency region of the gas can be found inside $\Delta \eta)$.

The use of the Generalized Moment Approach was found to provide very accurate estimates of cumulated distributions $g(k)$. The corresponding transmissivities can be evaluated using the usual formula encountered in $k$-distribution approaches (see for instance Ref. [3]), that provides an estimate of the band averaged transmission function $\tau^{\Delta \eta}(L)$ of a uniform gas path of length $L$ with spectral absorption $\kappa_{\eta}$ defined as:

$$
\tau^{\Delta \eta}(L)=\frac{1}{\Delta \eta} \cdot \int_{\Delta \eta} \exp \left(-\kappa_{\eta} \cdot L\right) d \eta
$$

as:

$$
\tau^{\Delta \eta}(L)=\int_{k_{\min }}^{k_{\max }} \frac{d g(k)}{d k} \cdot \exp (-k \cdot L) d k
$$

With 10 Generalized moments $(N=10)$, and 20 values of $k$ logarithmically spaced between $k_{\min }$ and $k_{\max }$, typical errors (defined as the absolute difference between the LBL reference data and its approximation) were found to be lower than $10^{-3}$ for transmissivities, over a wide 
range of test cases (in terms of temperature and gas path lengths). More details about the accuracy of the Generalized Moment Approach for application in radiative transfer can be found in Refs. $[9,10]$.

\section{A.2. CONNECTIONS BETWEEN CDPS AND SHIFTED-LEGENDRE}

\section{POLYNOMIALS.}

The aim of this section is to show that any polynomial at an integer order $N$ defined over the interval $[0,1]$ can be written as a sum of CDPs at the same order. Explicit formulas are given to write a polynomial in the CDP basis in terms of its coefficients in the canonical basis of $\mathbb{R}_{N}[X]$ (space of real polynomials with orders lower than $N$ ).

Let us consider an $N$-th order polynomial $P$, of the variable $X \in[0,1]$ :

$$
P(X)=a_{0}+a_{1} \cdot X+\ldots+a_{N} \cdot X^{N}
$$

We are seeking $N$ real numbers, $b_{0}, \ldots, b_{N-1}$ such that:

$$
P(X)=a_{0}+b_{0} \cdot \Phi_{0}^{(N)}(X)+\ldots+b_{N-1} \cdot \Phi_{N-1}^{(N)}(X)
$$

The derivative of $P$ with respect to $X$ is, from Eq. (A.8):

$$
\frac{d P(X)}{d X}=a_{1}+2 a_{2} X+\ldots+N a_{N} \cdot X^{N-1}
$$

and similarly, from Eq. (A.9):

$$
\frac{d P(X)}{d X}=b_{0} \cdot \frac{d \Phi_{0}^{(N)}(X)}{d X}+\ldots+b_{N-1} \cdot \frac{d \Phi_{N-1}^{(N)}(X)}{d X}
$$

Then, using the quasi-orthogonality property of the CDPs given by Eq. (A.5), we find that:

$$
\int_{0}^{1} X^{i} \frac{d P(X)}{d X} d X=b_{i}=\frac{1 \cdot a_{1}}{i+1}+\frac{2 \cdot a_{2}}{i+2}+\ldots+\frac{j \cdot a_{j}}{i+j}+\ldots+\frac{N \cdot a_{N}}{i+N}
$$

Eq. (A.12) provides an explicit formula to write any polynomial of $N$-th order in terms of CDPs (see Eq. (A.9)). It can thus be applied to shifted-Legendre polynomials (that are defined 
for values of $X$ inside the interval $[0,1]$ - more details about those polynomials can be found for instance in Ref. [20]). This shows that Eq. (10) can be written equivalently as Eq. (14-a).

\section{A.3. DEVYATOV'S GENERALIZED MOMENTS VS POLYNOMIAL CHAOS.}

The Generalized Moment and Polynomial Chaos approaches share many similarities: $1 /$ they are both based on a polynomial approximation of the output of a given model, 2/ the coefficients that appear in the polynomial developments are closely related to some moments of the model. The main difference between the two techniques is that:

- In Devyatov's approach [11,12], the objective is to study the transient response of a non-linear dynamical system. The solution of the problem is written as a sum of Cutteridge-Devyatov polynomials. The coefficients in the development (the generalized moments, that only depend on time) are estimated by using a BurmannLagrange series expansion.

- In PC methods, the objective is to characterize the output of a model subject to random inputs. This output is written as a sum of polynomials (whose mathematical form depends on the probability density function of the random input, as explained in Ref. [16]). The problem is then to estimate the coefficients that appear in the polynomial development, which are also generalized moments. Calculating those moments can be handled easily using Monte Carlo calculations.

The two approaches are thus fundamentally the same (approximation of the output of a model subject to some bounded stochastic or time dependent inputs by polynomials) but were originally proposed to treat different problems, in distinct communities. They can be summarized into what can be called the "Cutteridge-Devyatov Polynomial Chaos" (CDPC) method (another possibility would be to call them "spectral approximations" as they are both based on decompositions over sets of orthogonal or quasi-orthogonal polynomials - but this 
name would be confusing because most gas radiation models are already referred to as "spectral models", as soon as they account for the non-grey behavior of the radiative properties - the name "CDPC" avoids this possible misunderstanding and is fully justified, following the method proposed to introduce the technique detailed in this work).

One advantage of considering Devyatov's approach as a kind of Polynomial Chaos is that there are many works devoted to Polynomial Chaos (especially in English). The number of papers dedicated to Devyatov's moment approach is smaller, and most of them are in Russian. Furthermore, the full method seems to be easier to follow for people from the Radiative Transfer Community if the problem is formulated in terms of PC (because the starting point, which mainly consists in feeding a model with random variables, is very similar to the usual Monte Carlo technique, widely used in this community). Thinking in terms of (generalized) moments directly may be less intuitive.

Further work is required to develop the CDPC technique in a more general frame. 